\title{
Villemoustaussou (Aude) : Le Moulin Saint-Bernard
}

Tanguy Wibaut, E. Yebdri, Guilhem Sanchez

\section{Citer ce document / Cite this document :}

Wibaut Tanguy, Yebdri E., Sanchez Guilhem. Villemoustaussou (Aude) : Le Moulin Saint-Bernard. In: Archéologie du Midi médiéval. Tome 32, 2014. pp. 32-34;

doi : https://doi.org/10.3406/amime.2014.2065

https://www.persee.fr/doc/amime_0758-7708_2014_num_32_1_2065

Fichier pdf généré le 12/12/2019 


\section{Villemoustaussou (Aude) : Le Moulin Saint-Bernard*}

\section{Tanguy WIBAUT ${ }^{* *}$, Eric YEBDRI ${ }^{* *}$, Guilhem SANCHEZ ${ }^{* * * *}$}

\section{ELEMENTS GEOGRAPHIQUES ET TOPOGRAPHIQUES}

Cinq kilomètres au nord de Carcassonne, le site du Moulin Saint-Bernard se trouve aux abords sud du village de Villemoustaussou. Cette commune recouvre un territoire cantonné entre la plaine du Fresquel au sud et le Trapel, cours d'eau situé au nord-est. Le Moulin SaintBernard se situe sur un mamelon culminant à $108 \mathrm{~m}$ d'altitude au-dessus du village. La forte érosion des versants de cette éminence a fait que la grave et les cailloutis du substrat de molasse sont dans l'ensemble atteints à peu de profondeur. A l'ouest des parcelles sondées, le sol géologique se caractérise par des bancs de grès.

\section{CONDITIONS DE DECOUVERTE}

Un diagnostic archéologique a été réalisé en 2009 dans le cadre d'un projet de lotissement, les balcons $d u$ Moulin Saint-Bernard. L'intervention d'une semaine a permis d'explorer une surface d'environ 4 hectares. Aucune trace d'occupation n'était connue préalablement dans le secteur du Moulin Saint-Bernard.

\section{CHRONOLOGIE DU SITE}

Outre un paléochenal, le diagnostic a livré seulement deux structures excavées, apparemment isolées près de la limite ouest de l'emprise. De nature indéterminée, les deux structures semblent contemporaines, comme en témoignent leur comblement similaire et leur proximité (fig. 1). Le site ne semble pas occupé antérieurement ni après ces aménagements, mais cette chronologie reste fragile dans la mesure où l'établissement n'est que très partiellement délimité.

\section{PLAN ET STRUCTURE DE LA FOSSE 2201}

La fosse 2201, de plan rectangulaire, est orientée selon un axe nord-est/sud-ouest ; elle mesure 1,62 m. de longueur pour une largeur de $1,10 \mathrm{~m}$, soit une surface d'environ 1,80 $\mathrm{m}^{2}$; elle était creusée dans le substrat sur une profondeur maximale de $0,40 \mathrm{~m}$ et sous $0,65 \mathrm{~m}$ de terre arable (fig. 2). Cette faible profondeur d'enfouissement, ainsi que l'exiguïté du creusement, expliquent probablement l'absence de tout sol d'occupation à l'intérieur de la fosse. Dans l'ensemble les bords de cette excavation sont verticaux même si on perçoit sur les parois des irrégularités et de légers renflements, ainsi que des traces d'outils laissés lors du creusement. Le fond est plus ou moins plat, hormis vers la paroi est où le substrat remonte au contact de la paroi. Aux angles de l'excavation, légèrement arrondis, sont creusés quatre trous de poteau ; un seul de ces négatifs, à l'est, est circulaire tandis qu'à l'ouest, au sud et au nord les creusements sont plutôt rectangulaires, avec à l'intérieur l'empreinte circulaire des poteaux (fig. 3). Leurs diamètres varient entre $0,26 \mathrm{~m}$ et $0,44 \mathrm{~m}$, le plus profond atteint une profondeur de $0,40 \mathrm{~m}$ sous la base du creusement. Le comblement est homogène dans toute l'épaisseur du remplissage avec un sédiment argileux brun, dénotant un colmatage rapide. En dehors des trous de poteau, aucun aménagement particulier n'a été remarqué en fond de fosse.

Une seconde fosse oblongue, au comblement similaire et probablement contemporaine, FS 2202, se trouve à quelques mètres à l'est de la fosse 2201. Elle mesure $1,70 \mathrm{~m}$ sur $0,65 \mathrm{~m}$ pour $0,18 \mathrm{~m}$ de profondeur conservée. Malgré une fouille fine, elle n'a pas livré de mobilier. De contour irrégulier, elle ne présente aucun aménagement spécifique. Aucune autre structure de cette époque n'est présente dans l'emprise de l'opération ni connue aux alentours.

\section{FONCTION DE LA STRUCTURE EXCAVEE 2201}

Aucun élément ne permet d'avancer une hypothèse sur la fonction de cette structure. Malgré la particularité du plan rectangulaire à poteaux d'angles de FS 2201, qui évoque la morphologie d'une cabane à sol excavé, la surface extrêmement réduite ne milite pas en faveur

\footnotetext{
* Notice rédigée en 2014.

** Inrap Méditerranée

*** Inrap Méditerranée

**** Inrap Méditerranée
} 


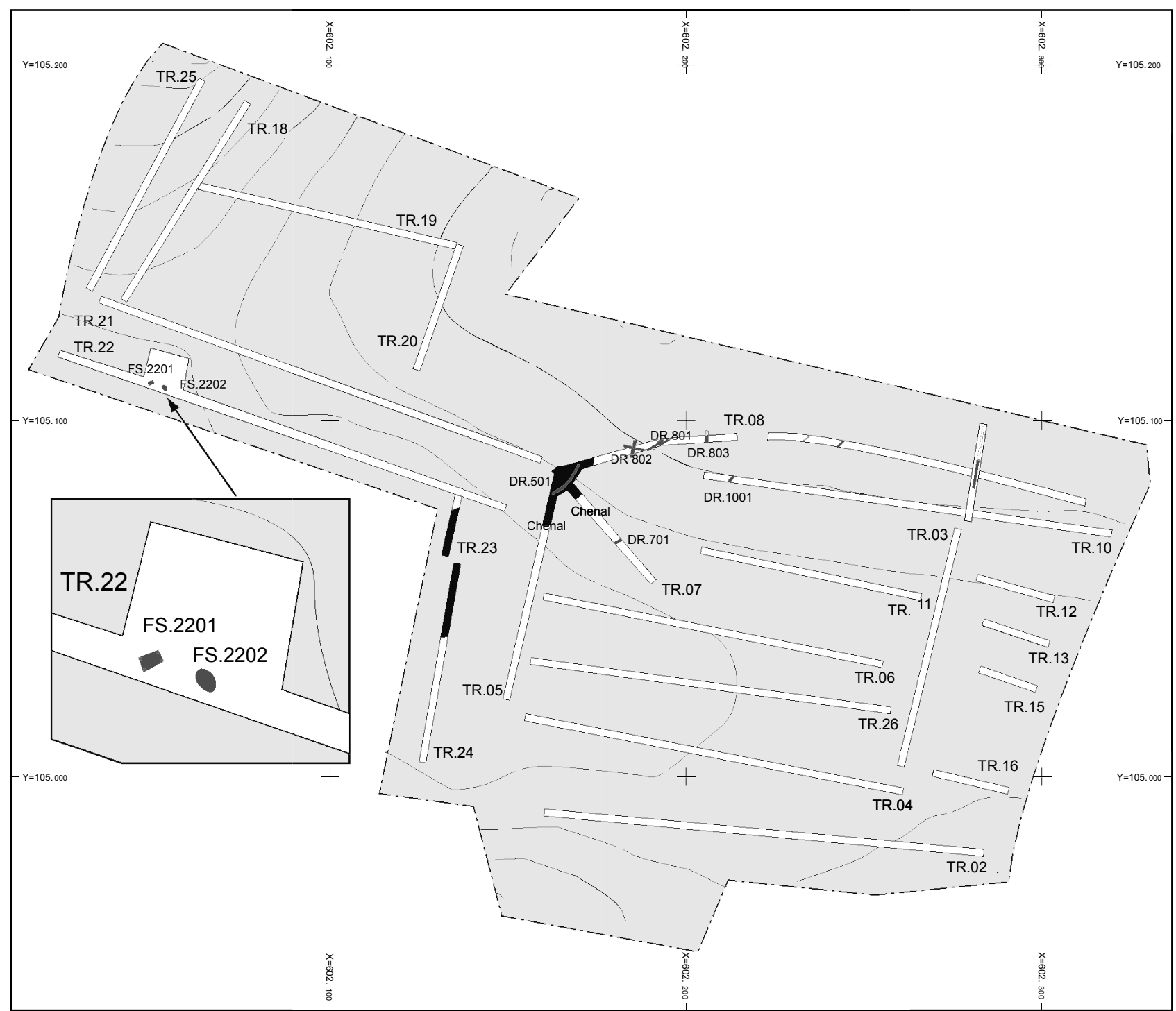

Fig. 1 : Villemoustaussou. Détail du plan du diagnostic présentant les structures FS 22.01 et FS 22.02.

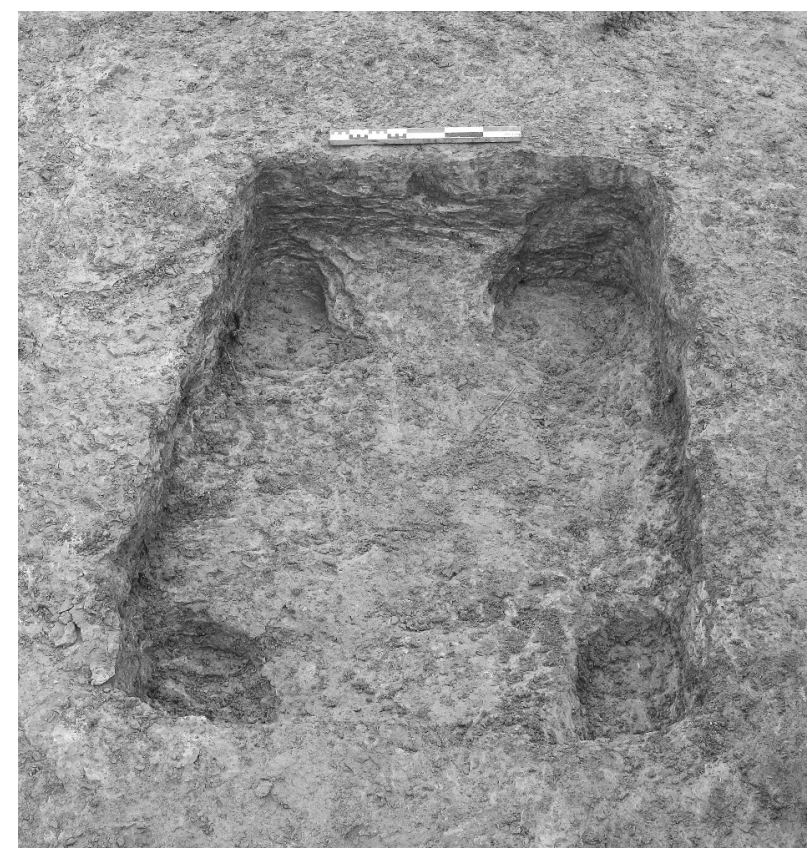

Fig. 2 : Villemoustaussou. La fosse 2201 vidée. d'une telle lecture. Les quatre poteaux ont probablement soutenu une superstructure, en bois. Ce type d'aménagement agricole ou artisanal appelle des parallèles connus sur des sites ruraux du haut Moyen Age en Ile-de-France, où l'on a proposé de voir des fosses d'ancrage de pressoir. De tels équipements sont connus dès l'Antiquité tardive mais ils mettent en jeu des excavations complexes, qui restent absentes à Villemoustaussou, peut-être en raison de l'arasement des niveaux (Deprataère-Dargery, Petit dir. 1993, 190 ; Couturier dir. 2003, 93-97).

\section{DATATION DE LA STRUCTURE EXCAVÉE}

Fouillée manuellement, l'excavation n'a livré qu'une dizaine de tessons de céramique, dont deux seulement ont pu être déterminés : un tesson de jatte non tournée CNT ROL C1, ainsi qu'un tesson de bol en céramique engobée CL ENG B6. Ces éléments permettent de dater le comblement du $\mathrm{IV}^{\mathrm{e}}$ siècle, grâce aux associations analogues observées notamment dans les contextes funéraires du Languedoc. 

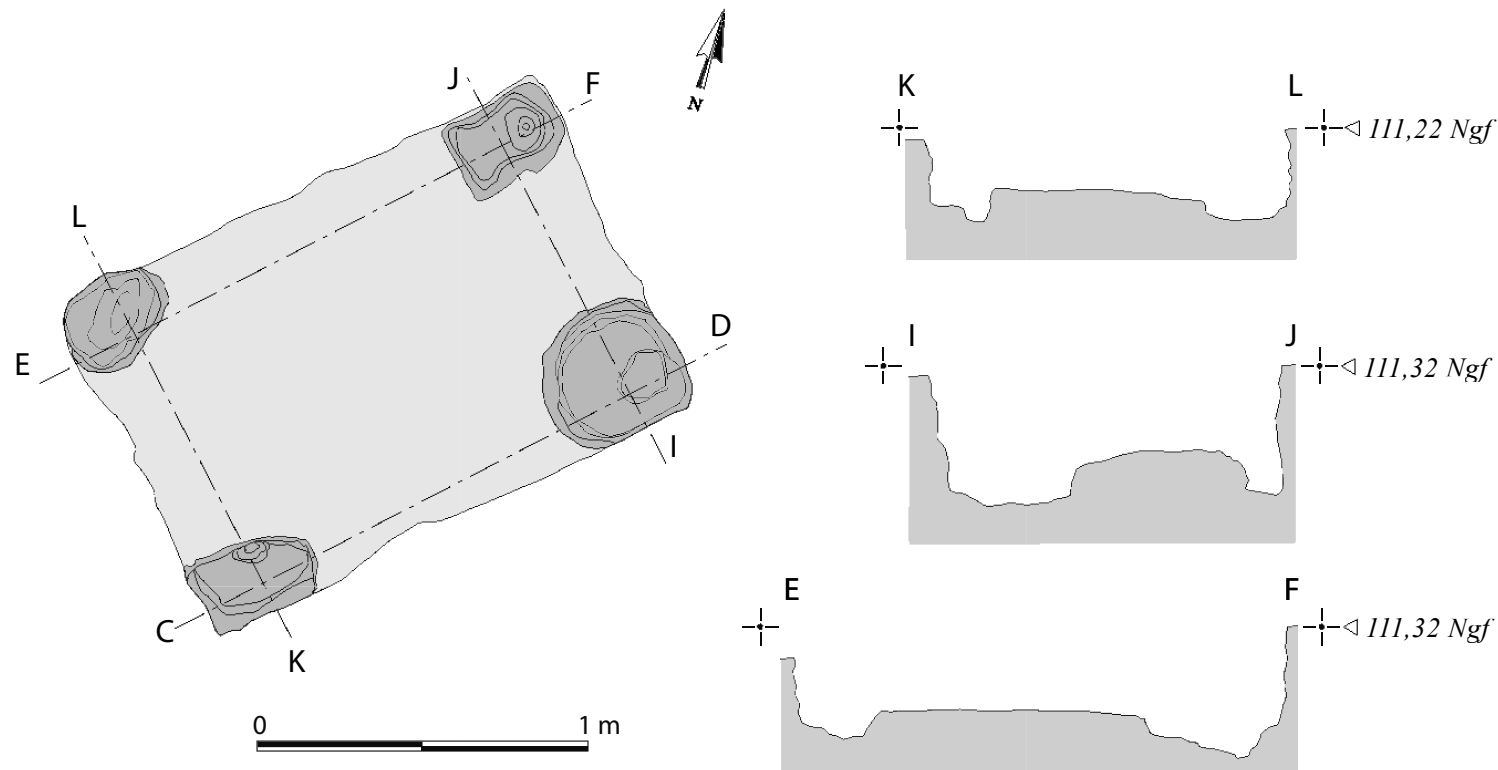

D
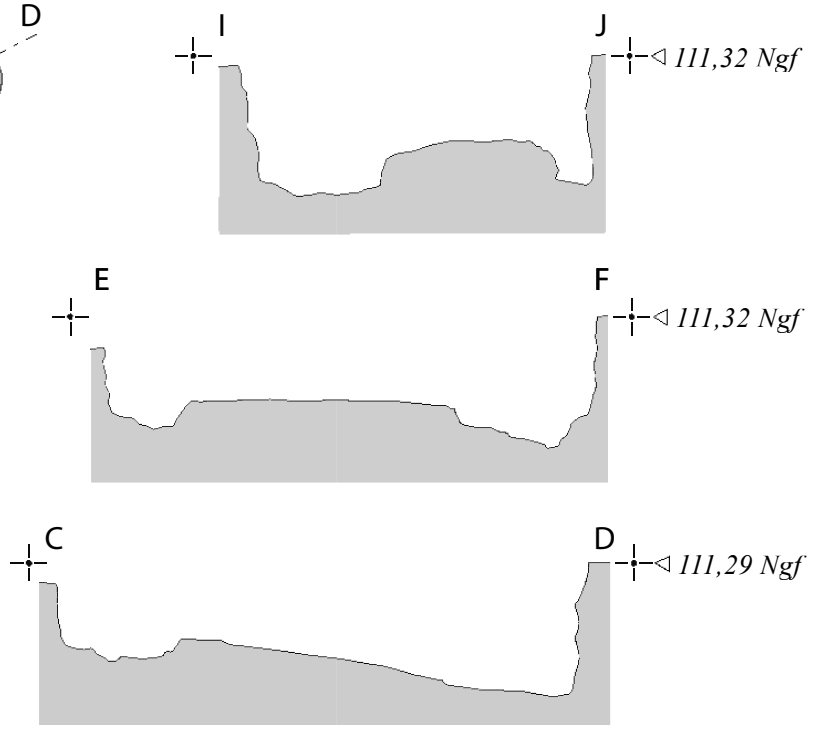

Fig. 3 : Villemoustaussou. Plan et coupes de l'excavation 2201.

\section{BIBLIOGRAPHIE}

Bilan Scientifique 2009 : Bilan scientifique de la région Languedoc-Roussillon, Montpellier, 2010, p. 55.

Deprataère-Dargery, Petit dir. 1993 : DEPRAETERE (M.), PETIT (M.) dir., L'Ile-de-France de Clovis à Hugues Capet, catalogue de l'exposition, Val-d'Oise, 1993, $343 \mathrm{p}$.
Couturier dir. 2003 : COUTURIER (D.) dir., Luzarches, le Bosquet : les installations agricoles et les niveaux de l'Antiquité tardive, Dioecesis Galliarum, Document de travail $\mathrm{n}^{\circ}$ 6, 2003, p. $77-106$. 